\title{
Las Experiencias Personales de los Niños como Pretexto para la Expresión Escrita
}

María Amalia Tarazona* Rocío Elizabeth Bonza Rojas**

Artículo de reflexión.

Fecha de Recepción: 28 octubre 2017.

Fecha de Aprobación: 2 marzo 2018.

\section{Resumen}

La labor pedagógica adelantada en el Colegio Técnico Municipal Simón Bolívar, Sede Jairo Aníbal Niño de la ciudad de Duitama, en los grados Primero y Segundo, ha llevado a que las educadoras identifiquen un problema álgido: La escritura centrada en el resultado y no en el proceso, desconociendo la verdadera función comunicativa de ésta. Por lo anterior, se busca contribuir al desarrollo de la competencia escritural de los estudiantes, de la mano de Emilia Ferreiro, Ana Teberosky, Celestin Freinet, Rubén Darío Hurtado, Daniel Cassany, entre otros, quienes ofrecen una mirada crítica y se constituyen en referentes teóricos. El trabajo se enmarca en el paradigma Cualitativo, con un enfoque Crítico Social, bajo una metodología de Investigación Acción Educativa; por lo cual se desarrolla en tres fases: Diagnóstico y exploración, Formulación de estrategias de acción, y, Análisis y valoración; se centra en el desarrollo de talleres que permiten generar espacios de significación, en donde la escritura cobra sentido, fundamentados en el conocimiento y las vivencias del niño. Es así como surge en él, la necesidad de escribir, la cual se pone de manifiesto en comportamientos, actitudes, preguntas; que llevan a la autoexigencia y reflexión sobre sus propios escritos.
Palabras
clave:
Habilidad escritural, expresión escrita, función comunicativa, proceso escritor natural, talleres pedagógicos.

\section{* Colegio Técnico \\ Municipal "Simón Bolívar" Sede "Jairo Aníbal Niño", Duitama. \\ amaliatarazona@yahoo.com ** Colegio Técnico Municipal "Simón Bolívar" Sede "Jairo Aníbal Niño", Duitama. \\ rossi20_04@hotmail.com}

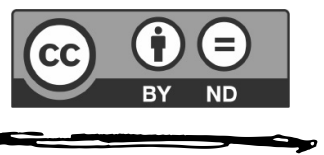


El hombre se caracteriza por ser un ser comunicativo por naturaleza, lo que consolida su dimensión de ser social.

\section{Introducción}

El hombre se caracteriza por ser un ser comunicativo por naturaleza, lo que consolida su dimensión de ser social. Cuando llega a desarrollar el habla para comunicarse, con el paso del tiempo encuentra que la transmisión oral, de generación en generación, si bien es un medio, no es suficiente para perpetuar su pensamiento, por lo cual, la escritura se convierte en el medio de interrelación con el mundo al difundir y plasmar sus ideas, su cultura, sus creaciones.

A este respecto, el niño, cuando empieza a tomar conciencia de su entorno, se adentra en el uso de las grafías, formas escriturales que le sirven para comunicar su pensamiento, sentimientos, deseos, expectativas, concepción de mundo, intereses, preguntas, respuestas, ganas de conocer lo desconocido...; a la vez que fortalece su autoestima, construye su identidad, toma sus propias decisiones y pone de relieve su autonomía...

Por su parte, paralelamente, el mundo de los adultos ha creado una cultura que aísla al niño de la atención que espera recibir para poder entablar su comunicación. Venimos de una cultura en la que muy pocas oportunidades, o ninguna, se han dado al niño; a éste se le ha considerado como un recipiente vacío al que hay que llenar, como alguien que tiene la obligación de aprender de los adultos y que no aporta nada frente al conocimiento de los demás. Así mismo, el niño, en su proceso de aprendizaje, usa sus grafías, escritura que no siempre es interpretada como tal por el adulto; a cambio de esto, le son impuestas formas gráficas que distan mucho de ser una verdadera comunicación; la escritura, entonces, pierde su carácter comunicativo y se convierte en algo tedioso, monótono y sin sentido; esta forma de "transmitir" la enseñanza de la "escritura" empieza a darse en el hogar (palitos y rayas) y continúa en la escuela, (planas); por otra parte, el adulto le da prioridad a la forma de la escritura, mas no al sentido de comunicación del niño, por lo que éste empieza a perder el gusto por la escritura y por la misma escuela, truncando así lo que debió ser un proceso de creación.

A partir de estos elementos se hace necesario hacer un alto en el quehacer pedagógico para resolver la pregunta: ¿Cómo se puede favorecer la producción de texto escrito en los estudiantes de los grados Primero y Segundo de la Sede "Jairo Aníbal Niño" del Colegio Técnico Municipal "Simón Bolívar", de Duitama? ante lo cual surge una propuesta que tiene como propósito favorecer la competencia escritural en el niño, mediante su propia expresión escrita; de esta forma, se pretende dar sentido a la apropiación de la escritura y rescatar el carácter comunicativo de la misma; para esto, es preciso tomar como referentes teóricos, diferentes experiencias e investigaciones de autores en el ámbito nacional e internacional, relacionadas con el desarrollo del proceso escritor natural del niño: Carminati, \& Wipan (2012), Jiménez (2000,2007); Rincón (1999); Hurtado, Serna \& Sierra (2003); Hurtado, Restrepo \& Herrera (2005 Febrero, Marzo), Quitian (2017); Jurado (1996), Freinet (1969, 1975), Cassany (1999,2011), autores 
que conciben la escritura como parte de un proceso complejo de naturaleza cognitiva, social, cultural y lingüística, a través de estrategias pedagógicas enmarcadas en situaciones funcionales, las cuales generan ambientes que favorecen dicho aprendizaje.

Por tanto, el maestro está llamado a conocer la forma como el niño construye su pensamiento escritor a partir de la recuperación de las experiencias personales del estudiante; se abre, entonces, un espacio para que éste conozca el placer de la expresión escrita: el poder de los signos gráficos, la creación de cuentos e historias, la posibilidad de comunicarse, todo lo cual genera un esfuerzo intelectual en él, que le permite acceder a niveles de abstracción cada vez más altos, logrando una escritura con sentido, agradable y, por ende, un aprendizaje para la vida. Estos planteamientos llevan a proponer situaciones pedagógicas a través del desarrollo de talleres, los cuales permitirán acompañar al niño en su proceso de construcción de aprendizaje y desarrollo de su competencia escritural, mediante la producción de escritos.

\section{Adentrándonos en el Mundo de la Escritura}

Para entender la forma como el niño elabora su sistema de escritura, se toman investigadores que revolucionaron la mirada que se tiene sobre el niño como sujeto del proceso comunicativo al asumir un papel activo en la construcción del conocimiento; tales autores, son, entre otros:

Tabla 1.

Fundamentos teóricos

\begin{tabular}{|c|c|}
\hline AUTOR & TESIS O PLANTEAMIENTO \\
\hline $\begin{array}{l}\text { MABEL CARMINATI } \\
(2012)\end{array}$ & $\begin{array}{l}\text { I La escritura constituye un ejercicio mental, que estimula constantemente la } \\
\text { actividad neuronal que, a su vez, cambia permanentemente la estructura del } \\
\text { cerebro (plasticidad), ya que adquirir este proceso implica la utilización de todas } \\
\text { las estructuras mentales de aprendizaje superior (percepción, memoria, aprendizaje) } \\
\text { funcionando de manera conjunta y coordinada. A medida que va creciendo el } \\
\text { niño, el lenguaje egocéntrico que utiliza se va volviendo más simbólico, empieza a } \\
\text { utilizar sus garabatos para expresarse con los demás, pero la necesidad de expresión } \\
\text { va aumentando, y es ahí donde a través del dibujo el niño intenta reflejar lo que } \\
\text { piensa, teniendo plena conciencia de su función comunicativa. Esta expresión } \\
\text { artística se transforma, pasando por fases progresivas que lo llevarán a la adquisición } \\
\text { de la escritura convencional. }\end{array}$ \\
\hline $\begin{array}{l}\text { ANA TEBEROSKY Y } \\
\text { EMILIA FERREIRO }\end{array}$ & $\begin{array}{l}\text { Los niños para aprender a leer y escribir, tal y como sucede con cualquier } \\
\text { construcción cognitiva, pasan por diferentes niveles, formulando distintas hipótesis } \\
\text { respecto a cómo se escribe, que van mejorando a medida que las ponen a prueba } \\
\text { en distintos actos de escritura y se enfrentan a distintos conflictos cognitivos. Este } \\
\text { proceso de construcción del sistema de escritura tiene un ritmo individual y está } \\
\text { condicionado, entre otras cuestiones, por el tipo de interacciones que el niño tenga } \\
\text { en los distintos contextos letrados, por el interés y la motivación ante la propuesta } \\
\text { escrita, la autonomía emocional para resolver actos de escritura. }\end{array}$ \\
\hline
\end{tabular}


YANICE VIEGAS (2015) Afirma que el proceso escritural es una construcción cognitiva y social por lo cual, el niño inicia su escolaridad con conocimientos y vivencias, en cuanto a escritura se refiere; se hace necesario, entonces, poner en juego estos saberes para favorecer y continuar su avance natural, permitiéndole la experimentación libre sobre las marcas escritas; es aquí donde cobra importancia la producción, ya que da la oportunidad al niño de interactuar con la escritura en situaciones que le permiten analizar, contrastar, reflexionar, inferir y verificar sus propios puntos de vista sobre el sistema escrito.

GLORIA RINCÓN Y Resignifica la escritura como proceso de naturaleza cognitiva, social y lingüística, OTROS (1999) con función real de comunicación, en la que se hace necesario, entonces, poner en juego saberes que trae el niño, acerca de la escritura, para favorecer y continuar su avance natural, permitiéndole la experimentación libre sobre las marcas escritas; es aquí donde cobra importancia la producción, ya que da la oportunidad al niño de interactuar con la escritura en situaciones que le permiten analizar, contrastar, reflexionar, inferir y verificar sus propios puntos de vista sobre la misma.

RUBEN DARIO Presenta la escritura como habilidad comunicativa determinada esencialmente HURTADO, SERNA Y por el pensamiento y el lenguaje en las que se privilegia la comprensión sobre SIERRA (1989) la mecanización, ya que es concebida como un proceso de construcción de significados.

RUBEN DARIO La metacognición, entendida como la capacidad de autorregular los procesos de H U R T A D O , aprendizaje, que le permiten acceder a niveles de cualificación más altos; es allí RESTREPO $\quad Y$ donde juega un papel fundamental la producción de escritos, de modo que la HERRERA (2003) escritura se convierte en un medio activo para desarrollar las habilidades cognitivas en los niños, al lograr un mayor grado de conciencia sobre el texto.

RUBEN DARIO Identifica diversas formas de corrección de escritura entre pares, donde se busca H U R T A D O , que los niños tomen conciencia de cómo escriben, $y$, a partir de ahí, emprender un RESTREPO Y proceso metacognitivo a través de la "Confrontación Pautada"

HERRERA (2003)

FABIO JURADO Muestra cómo el análisis escritor, desde la perspectiva semiótica, puede contribuir a (1992) la formación de competencias lecto-escritoras, y cómo la escuela es eje fundamental, al cambiar su visión tradicional por una escritura como proceso ordenador y cohesionador de los saberes al fomentar la producción de escritos y la reescritura de los mismos por parte de los estudiantes.

CELESTIN FREINET Otorga, tanto al niño como al maestro, un papel dinámico, por un lado, el niño es agente activo en la construcción del conocimiento, por el otro, el docente se convierte en el orientador del proceso educativo; de esta manera, se formula una relación educativa horizontal y de apoyo mutuo. Para ello, crea una serie de técnicas, que surgen en su larga experiencia docente, que se basan en el contexto social del estudiante, en sus intereses y necesidades, a la vez, que se resalta el trabajo colaborativo del grupo.

NEGRET Y OTROS Resalta la importancia de fortalecer la relación del niño con la escritura, hacerla (2011) importante y con sentido; libre de modelos ideales constrictores que inhiban su proceso escritural, por ello, la corrección por parte del adulto solo debe darse cuando el niño ha empezado a producir frecuentemente y con su propio estilo.

DANIEL CASSANY Ofrece una didáctica de la composición escrita en la que se destaca el carácter comunicativo funcional de la actividad lingüística escrita, bajo una práctica frecuente en el aula, a "hablar" acerca de lo que se escribe y a compartir y confrontar el escrito con los pares. 
Los planteamientosanteriores evidencian que el niño debe ser protagonista en su proceso de adquisición y aprendizaje del lenguaje escrito, con el que puede comunicarse, expresarse, desarrollar su autonomía y poner en juego su conciencia. Sin embargo, en la práctica cotidiana, el niño es el gran ausente en dicho proceso, así el discurso de la escuela, al respecto, indique lo contrario.

\section{Metodología}

Con miras a contribuir al análisis y mejoramiento de la práctica pedagógica se acude al paradigma Cualitativo con un enfoque Crítico Social; se basa en un tipo de Investigación-Acción Educativa, la cual es definida por Elliot, J (1993) como: "Un estudio de una situación social con el fin de mejorar la calidad de la acción dentro de la misma” (p. 88), en este caso, desde el contexto educativo en donde se labora; por cuanto se pretende realizar una investigación de carácter formativo, ya que parte del análisis de la realidad, las necesidades, las expectativas, las potencialidades y el conocimiento del niño, a través de diferentes medios; para luego identificar problemas que obstaculizan su formación integral, y, así plantear preguntas en torno al mismo, en este caso, la necesidad de favorecer la competencia escritural de los niños; de esta forma diseñar acciones que se construyen desde y para la práctica; en busca de contribuir al fortalecimiento de los procesos comunicativos de los niños mediante la producción de texto escrito.

Cabe decir que, la población hace parte del Colegio Técnico Municipal "Simón
Bolívar", el cual se encuentra ubicado en el perímetro urbano del Municipio de Duitama, Departamento de Boyacá; es una Institución de carácter oficial que ofrece los niveles de Preescolar, Básica y Media Técnica, en las modalidades Presencial y Semipresencial; posee una sede central y cuatro sedes de Educación Básica Primaria.

La investigación se desarrolla en la Sede "Jairo Aníbal Niño", la cual se encuentra ubicada en el barrio "Juan Grande"; atiende a estudiantes en los niveles de preescolar y de básica primaria. La unidad de análisis corresponde a los grados primero y segundo, conformada por 46 estudiantes, en edades que oscilan entre los 6 y los 12 años; los escolares hacen parte de una comunidad cuyo nivel socioeconómico corresponde a los estratos uno y dos, pertenecientes a familias extensas las cuales están integradas por padres, abuelos, tíos, primos y otros parientes consanguíneos; también se encuentran algunas familias monoparentales, es decir, conformadas por uno de los padres y sus hijos; hay presencia de familias nucleares, aquellas constituidas por los padres y sus hijos; también hay tendencia a la disolución familiar, por lo que se ha venido incrementando la presencia de familias compuestas, en las cuales, uno o ambos miembros de la actual pareja tiene uno o varios hijos de uniones anteriores. Se manifiesta un alto índice de desempleo en los padres, reflejado en el trabajo informal de los mismos; por esta razón, en muchos casos, los niños son dejados solos o al cuidado de terceros, por lo que éstos no encuentran interacción comunicativa efectiva en casa.

\footnotetext{
La investigación se desarrolla en la Sede "Jairo Aníbal Niño", la cual se encuentra ubicada en el barrio "Juan Grande"; atiende a estudiantes en los niveles de preescolar y de básica primaria.

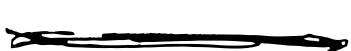


Cabe anotar, que el presente trabajo sólo es utilizado con fines académicos e investigativos y la información aquí obtenida es confidencial. Al ser un estudio de tipo cualitativo no se realiza ningún tipo de intervención a los participantes; de igual forma, los padres de familia y los estudiantes, conocen la finalidad de la información recogida; para ello se solicita el consentimiento en el caso que se requiera, según las actividades propuestas, de otro lado se hace énfasis en la equidad y en la no discriminación de grupos o personas participantes.

Ahora bien; se asumen tres fases en el diseño de la Investigación Acción Educativa, las cuales funcionan de manera interdependiente, a saber:

Fase de diagnóstico y exploración. Esta etapa consiste en el conocimiento de la realidad educativa, recolectando información pertinente, para identificar una situación problema, abarcando tanto sus manifestaciones como las consecuencias y repercusiones del mismo. Para ello, se utilizaron las siguientes técnicas e instrumentos en la recolección de información:

Observación directa. Tal como lo dice Hernández (2010), "No es mera contemplación("Sentarse a ver el mundo y tomar nota"), implica adentrarnos en profundidad a situaciones sociales y mantener un papel activo, así como una reflexión permanente. Estar atento a los detalles, sucesos, eventos e interacciones" (p.411). Es por ello que se realiza la observación directa en la escuela, con el propósito de analizar actitudes y comportamientos del niño al enfrentarse al acto de escribir, así como, los textos creados por los niños, de manera espontanea, que permiten identificar las hipótesis que de escritura tienen.

Cuestionario cualitativo. Éste fue aplicado a docentes de los grados Pre-escolar, Primero y Segundo de la Institución Educativa para conocer la concepción que de escritura se tiene, las estrategias empleadas en el proceso de adquisición de la misma y el papel que juega la producción de escritos en el aula.

Revisión de documentos. Con el fin de identificar las debilidades presentadas por los estudiantes en el área de Lenguaje, se procede a la revisión y análisis de pruebas externas, tales como: Histórico de resultados Prueba Saber, grado tercero, años 2015 y 2016, y, resultados Pruebas Saber 2016, Grado tercero.

De igual forma, se analizan los Derecho Básicos de Aprendizaje (DBA), primera y segunda edición, en el área de lenguaje, por cuanto son un conjunto de saberes y habilidades fundamentales en el proceso de formación de los estudiantes los cuales permiten, a la vez, plantear elementos para construir rutas de aprendizaje.

Revisión bibliográfica. Antela necesidad de profundizar en el conocimiento del proceso escritor natural del niño, se lleva a cabo una revisión bibliográfica de autores e investigadores, los que se constituyen en referentes de la escritura como procesos de construcción $\mathrm{y}$ desarrollo del pensamiento. 
Fase de formulación de estrategias de acción. Teniendo en cuenta el análisis y reflexión que se lleva a cabo en la etapa diagnóstica y atendiendo a la revisión bibliográfica, se procede a formular estrategias de acción, las cuales se centran en talleres pedagógicos que superan la simple aprehensión de conocimientos o el desarrollo de actividades desarticuladas, para llegar a vivir una experiencia de aprendizaje que rescate el valor comunicativo de la escritura con sentido para el niño, que responda a sus necesidades e intereses, le otorgue valor y significado a la vida escolar, a la vez que se apoya en el trabajo colaborativo para reconocer la importancia del otro, tal como lo refiere Egg (1993), al definirlo como "Una forma de enseñar y, sobre todo aprender, mediante la realización de algo que se lleva a cabo conjuntamente. Es un aprender haciendo en grupo" (p. 8).

Estos talleres pedagógicos, integran al estudiante y al docente en una dinámica que favorece el uso y la apropiación del lenguaje escrito en situaciones reales de comunicación, al poner en juego los conocimientos previos del niño y, así, ofrecer experiencias significativas que le permitan movilizar sus ideas o hipótesis que del sistema escrito tiene; de la misma forma, se propicia un ambiente para que el niño aprenda con el otro, es decir, aprender "juntos". Para ello, se incentiva al niño a escribir desde el primer día de clase, rescatando momentos importantes y significativos, y que se convierten en material de comunicación real; a partir de esto, se diseñan situaciones que facilitan la reflexión sobre el sistema escrito.

Dichas situaciones llevan a concertar tópicos que se desarrollan en los siguientes talleres:

\section{Taller 1. Correo de los sentimientos.}

Con el desarrollo de este taller se busca propiciar espacios que permitan intercambiar emociones y sentimientos entre padres e hijos, a través del lenguaje escrito, cumpliendo, así, su función comunicativa, a la vez que se convierte en el momento propicio para incentivar al niño a escribir. Es así como, en un primer momento se realiza una actividad de sensibilización, dirigida a los padres, en el que se da a conocer el proceso por el cual el niño adquiere la escritura, a través de mensajes elaborados por los niños con anterioridad, para que los padres den respuesta y sea éste el pretexto para incentivar la comunicación, por lo que surge "El buzón de los sentimientos". En un segundo momento, los estudiantes escriben una nueva carta, atendiendo a un proceso de planificación del escrito que se define en interrogantes como: ¿Qué va a escribir?, ¿Para quién va a escribir?, y ¿Qué va a expresar?

\section{Taller 2. Mi nombre me hace una} persona importante. Al utilizar el nombre como estrategia didáctica, se accede a niveles de construcción escritural más altos, con lo cual se favorece la función comunicativa a través de la creación de textos escritos con significado. Para ello, se hace necesario que cada niño conozca la historia de su nombre, a través de actividades que van desde la elaboración conjunta (estudiantes, docentes) de una entrevista dirigida a los padres, la cual 
Se trabaja la unión del grupo y las habilidades de comunicación, lo cual mejora significativamente la convivencia dentro del aula, ello, en parte, debido a que los textos se corrigen en forma colectiva le permite indagar acerca de su nombre, hasta dinámicas variadas que le llevan a expresarse de forma oral y escrita. Para la elaboración de los escritos se tiene en cuenta el modelo de composición, citado por Hurtado (2005), el cual refiere los proceso cognitivos de Planificación, Textualización y Revisión; y que permiten a su vez, traducir las ideas, pensamientos, sentimientos, impresiones que posee el sujeto, en un discurso escrito coherente, en función de contextos comunicativos y sociales determinados.

Taller 3. Una fecha especial. Tiene como propósito promover la escritura de textos con sentido para el niño, a través del rescate de sus vivencias personales, sus necesidades e intereses, que favorezcan la contextualización del aprendizaje. Por esto se recurre a la elaboración de cuadrogramas, maqueta que muestra en primer plano unos personajes y/o elementos y en el fondo una representación del lugar donde están inmersos para recrear una escena, que ilustran una fecha especial, los cuales son socializados, de manera oral, con los compañeros. Seguidamente se desarrolla el momento de construcción de escritos, en el que el docente juega un papel primordial, donde incentiva al análisis del texto producido, a través de la Confrontación Pautada.

Taller 4 Texto libre. Su propósito es dar sentido de comunicación a la palabra escrita, mediante la utilización de la técnica del "Texto Libre", propuesta por Célestin Freinet. Es, entonces, necesario seguir ciertos pasos que favorecen su función pedagógica: La escritura del texto, por parte del niño, cuando siente la necesidad de expresarse; lectura en común en la que el texto se comparte con los compañeros; por tanto, exige un trabajo de lectura, entonación, modulación de la voz, interpretación y expresión oral ante el grupo de pares; elección de texto leído, aquí surge un trabajo auténticamente democrático, ya que mediante votación, los niños eligen entre los textos que se han leído, aquel que se trabajará en clase, favoreciendo la participación y toma de decisiones de manera autónoma; el texto elegido es escrito en el tablero, para que todos los niños tengan acceso al mismo, se comienza a perfilar a nivel gramatical, ortográfico...adecuándolo a la situación y a lo que el autor quiere expresar. "Se trabaja la unión del grupo y las habilidades de comunicación, lo cual mejora significativamente la convivencia dentro del aula, ello, en parte, debido a que los textos se corrigen en forma colectiva" (Freinet, 1975); a continuación el autor vuelve a escribir el texto, éste es fotocopiado y se distribuye en el grupo, para que cada uno lo ilustre y se apropie del mismo; por último, los textos elaborados por los niños son recopilados y compartidos con estudiantes de otros grados de la institución, de esta manera se promueve, no solo que el niño escriba, sino que escriba para alguien.

Taller 5. Cuando grande, quiero ser. Este taller permite conocer intereses, necesidades y sueños del niño que se convierten en motor de expresión y en material de comunicación, a la vez que le da la posibilidad de ubicarse en situaciones funcionales de escritura ,y , 
asumir retos intelectuales para construir su propia narrativa sobre el mundo, a través de sus escritos. Para su desarrollo se utilizan videos educativos, juegos interactivos, mímica y "Veritek" (juego de autocorrección presentado por la Corporación para la Inclusión y la Diversidad, CORPIDI, el cual promueve el desarrollo del razonamiento, el análisis, la discriminación, comprensión de lectura y lenguaje). En cuanto a la producción de texto escrito se emplean estrategias metacognitivas, las cuales posibilitan la reflexión a través de preguntas problematizadoras, que llevan al niño a analizar, reflexionar, contrastar, verificar y cuestionar sus propios escritos.

Análisis y valoración. Esta fase implica un "comportamiento reflexivo" de toma de decisiones justificadas y una responsabilidad directa con el contexto educativo en lo referente a los hallazgos del trabajo realizado. Al ser un proceso cíclico y en espiral, la reflexión se da desde un comienzo, haciendo parte de cada uno de los aspectos trazados; permitiendo así, comprender de forma integral la naturaleza del problema, objeto de estudio, y de esta forma conocer el impacto pedagógico alcanzado, a través del desarrollo de la propuesta.

Para ello, se emplea la matriz de análisis que permite la reducción de los datos obtenidos en cada una de las fases, a partir de la cual se genera las categorías de adecuación, cohesión y coherencia. Dichas categorías son analizadas en los propios escritos de los niños, a través de rejillas, las que facilitan transformar la visión lineal en una visión total e interrelacionada, y dan cuenta del avance en relación con su competencia escritural.

\section{Resultados}

A continuación, se presentan los resultados, atendiendo a los aportes dados por los antecedentes y referentes teóricos, estudiados para tal fin y a la apreciación personal, a partir de la información obtenida en la cada una de las fases.

Fase 1. Diagnóstico y exploración. El niño antes de ingresar a la escuela ha construido una serie de conocimientos sobre la lengua escrita, sus marcas y trazos son muestra de la forma como éste concibe la escritura. Por ello, el docente debe conocer el proceso escritural que el niño ha iniciado, tal como como lo presentan en sus investigaciones Ana Teberosky y Emilia Ferreiro, para quienes las representaciones gráficas de los niños son los indicadores del tipo de hipótesis que ellos elaboran. Identificar estos modos de organización implica reconocer que existe una psicogénesis del principio alfabético de escritura, ya que el proceso escritor del niño se va consolidando de acuerdo con su intencionalidad comunicativa particular; el maestro debe contribuir a su avance, propiciando situaciones que le permitan al niño enfrentar el acto de escribir, con lo cual se moviliza la construcción del conocimiento a partir de sus intereses y la integración de la realidad social que vive al contexto escolar; de allí radica la importancia de la producción textual escrita. 
La apropiación de la competencia escritural por parte del niño se ve obstaculizada por la forma como el docente concibe la escritura y por ende, asume su "enseñanza" centrada en la forma de las letras, palabras y oraciones, con lo que se coarta las posibilidades del niño y su interés por seguir construyendo su proceso escritor natural; así lo afirma Rincón, et al. (1999), al resignificar la función de la escritura. A esto se suma el hecho de querer homogeneizar la forma de aprender, sin tener en cuenta que ésta es producto de una serie de procesos cognitivos propios de cada ser, determinados por el contexto en el cual se desenvuelve.

A pesar de que los Derechos Básicos de Aprendizaje (DBA), han sido revisados, reevaluados y rediseñados, presentando cambios significativos en cuanto a la concepción del sistema escrito, ya que se da mayor importancia al proceso de construcción del mismo; muchas veces no son llevados a la práctica en la escuela por la concepción que de ésta tiene el docente; es por ello que se limitan las posibilidades del niño a, la vez que se le impide la discusión, argumentación y libertad, con lo que la escuela no respeta las diferencias, trayendo consigo una educación que uniforma el pensamiento.

Fase 2. Formulación de estrategias de acción. A través del desarrollo de talleres, se encontraron los siguientes resultados:

Con el desarrollo de cada una de las actividades, se evidenció la necesidad del niño de escribir, la cual se puso de relieve mediante comportamientos, actitudes, preguntas; auto exigiéndose y reflexionando sobre sus propias producciones escritas, rescatando la verdadera función de la escritura, la comunicación; por tanto, la tarea de escribir debe estar relacionada con temas que tengan que ver con las vivencias y experiencias presentes en la vida del niño y en el contexto en que éste se desenvuelve, solo así se podrá dar sentido y significado a la escritura.

A través de la estrategia de la Confrontación, pautada y colectiva, el niño analizó acerca de su escrito, por medio de preguntas que se convirtieron en situaciones problema y que requirió de un ejercicio mental para su solución, al utilizar el conocimiento que ya posee de la escritura.

Así mismo, y con relación a la reescritura de textos, ésta permitió facilitar la reflexión en los niños sobre el sistema de escritura, dando un valor pedagógico al "error", pues más que una recriminación o descalificación, es una oportunidad de aprendizaje; como lo manifiesta Ferreiro (2002) "es convocar a los niños a la reflexión y a la búsqueda de alternativas de solución que genera la tarea de comprender y producir textos con sentido" (p. 18)

De otra parte, el trabajo en grupo permitió al niño convivir con la diferencia y aprender la importancia de ayudar y ser ayudado, a la vez que dinamizó intercambios personales, en la medida que cada integrante mostró lo que sabe e hizo su aporte al grupo; de igual forma, se favoreció el trabajo colaborativo, ya que el niño comprendió que pertenecer a un grupo significa 
ser parte activa de él trabajando todos mancomunadamente para dar solución a una situación, y no al hecho de imponer su criterio sin tener en cuenta la opinión de los demás.

Durante todo el proceso, en el cual los niños pusieron en marcha, de manera organizada y productiva, su dinamismo natural, manifestado en su deseo y creatividad al escribir; fue fundamental la orientación de las docentes, quienes a través de variadas actividades, promovieron la reflexión, la discusión y el cuestionamiento permanente; lo que viabilizó espacios de comunicación efectiva y eficiente.

Si bien, cada escrito responde a las características individuales del niño, se pudoevidenciarquelos textos producidos se hicieron más comprensibles, reflejado en: Relación entre emisor, destinatario, contexto y enunciado; organización del escrito al ordenarlo de manera lógica y comprensible para su lector; utilización de algunos recursos para encadenar las distintas ideas del escrito, entre ellos conectores, espacios y separación de enunciados.

\section{Conclusiones}

Para revalidar la función comunicativa y favorecer la competencia escritural del niño, a través de la producción de sus propios escritos, es necesario entender que:

- La escritura es un proceso de construcción de pensamiento, un evento de significación e interacción comunicativa mediada por un proceso activo de carácter cognitivo, social y emocional, tal como, lo afirman Ana Teberosky y Emilia Ferreriro, al plantear la psicogénesis de la escritura, como resultado de sus investigaciones. Es por esto que, con el desarrollo de la propuesta se da continuidad al proceso escritor natural del niño, el cual ha iniciado mucho antes de ingresar a la escuela, se revalida la verdadera función comunicativa de la lengua escrita, para que a través de esta se contribuya a la construcción de conocimiento.

- Es fundamental que el docente conozca la forma como el niño adquiere el proceso escritor natural, esto implica que desaprenda la forma como ha venido enseñando tradicionalmente la escritura, ya que durante mucho tiempo ha estado en una zona de confort que ha dificultado el desarrollo de la competencia escritural.

- Al hacer de la enseñanza de la escritura una actividad contextualizada, donde el niño sea el centro de la práctica pedagógica y ésta responda a sus necesidades, intereses y expectativas, se generan aprendizajes más duraderos y significativos.

- El maestro, debe entender que solo se enseña a escribir cuando es el niño ese ser capaz de producir su propio pensamiento, de comunicar sus ideas, necesidades y sentimientos a través de la palabra escrita, lo que lleva a repensar la práctica pedagógica de manera contextual e integral, solo así, se genera un aprendizaje eficaz y eficiente, en una palabra, significativo; en concordancia con los 
aportes dados por Celestín Freinet, quien plantea el uso de estrategias que permiten al niño poner en juego sus conocimientos y acceder a otros niveles de construcción y comprensión en cuanto a lengua escrita se refiere.

- Si se considera la escritura como un instrumento de comunicación, de representación y de generación de pensamientos, es decir, un proceso de construcción de significados, como lo manifiesta Rubén Darío Hurtado; la enseñanza de la escritura se convierte en una actividad contextualizada, donde el niño es el centro de la práctica pedagógica y ésta responde a sus necesidades, intereses y expectativas, generando aprendizajes más duraderos y significativos.

\section{Referencias}

Arellano, M. (2006). Proyecto de innovación: Técnicas Freinet, aplicación del texto libre en primaria. Tesis de pregrado. Universidad Pedagógica Nacional. México

Carminati, M., \& Wipan, L. (2012). Integrando la neuroeducación la aula. Buenos Aires: Bonum.

Cassany, D. (1999). Construir escritura. Madrid: Ed Paidos.

Cassany, D., Luna, M. \& Sáenz, G. (2011). Enseñar lengua. Barcelona: Ed Graó.

Consejo nacional de Fomento educativo. (2011). Aprendo con el nombre. Guía para enseñar a leer a partir del nombre propio. México D.F.

De Acevedo, A. (2014). Cómo funciona el cerebro de los niños?. Bogotá D.C: Grijalbo.

Egg, E. (1993). El taller una alternativa de renovación pedagógica. Buenos Aires: Magisterio del Río de la Plata.

Freinet C. (1969). Técnicas Freinet en la escuela moderna. México: Siglo XXI editores.

Freinet C. (1975) Método tradicional de lectura. Barcelona: editorial LIIA.

Freinet E. (1978). Trayectoria de Célestin Freinet. España: Payot.

González M. (1998). La pedagogía de Celestín Freinet: Contextos, bases teóricas, influencias. Madrid: Ministerio de educación y ciencia

Gurdián, A (2007). Paradigma cualitativo en la investigación Socio-educativa. San José, Costa Rica: Colección Investigación y Desarrollo Educativo Regional (IDER)

Hurtado, V., Serna, D., \& Sierra, M.( 2003). Lectura y escritura en la infancia. Estrategias pedagógicas para facilitar su construcción: Fundación Colombia. 
Hurtado, V., Restrepo A., \& Herrera, O. (2005). Escritura reflexiva. Una propuesta didáctica para la básica primaria. Universidad de Antioquia, Facultad de educación: Centro de investigaciones educativas pedagógicas, Medellín.

Hurtado, R. (2010). La confrontación pautada: una estrategia didáctica para dinamizar los procesos de constucción de la lectura y la escritura en los niños de educación preescolar y básica primaria. Universidad de Antioquia, Facultad de educación, Medellín.

Jiménez, V, C. (2000). Cerebro Creativo y Lúdico. Bogotá: Editorial Magisterio.

Jiménez, V, C. (2007). Neuropedagogía, lúdica y competencias; Nuevos métodos desde la neurociencia para escribir, leer, hablar, estudiar y utilizar pedagógicamente las inteligencias múltiples. Bogotá D.C: Editorial Magisterio.

Jurado, F.(1996). Los procesos de la escritura. Hacia la producción interactiva de los sentidos. Santafé de Bogotá D.C: Cooperativa editorial Magisterio.

Ministerio de Educación Nacional (2006). Estándares básicos de competencias en lenguaje, Matemáticas, Ciencias y Ciudadanas. Guía sobre lo que los estudiantes deben saber y saber hacer con lo que aprenden. Santafé de Bogotá.

Ministerio de Educación Nacional (2016). Derechos básicos de aprendizaje. Santafé de Bogotá.

Quitián, S. (2017). Oralidad y escritura: campos de investigación en pedagogía de la lengua materna. Praxis e Saber, 8(16), 133 -153. https://doi. org/10.19053/22160159.v8.n16.2017.6181

Rincón, G., Bustamante, G., Pérez de Rodríguez, D., \& Castro, M. (1999).La enseñanza de la lengua escrita. Desde pre-escolar hasta tercer grado de educación básica. Santafé de Bogotá: Arango editores.

Viegas, J. (2015). Didáctica socio interaccionista. Módulo de orientación teórico-práctica lenguaje. Cali: Fundación transforma por una educación emancipadora. 